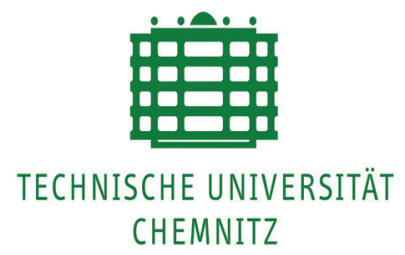

Roland Pässler

Alternative Way for Detecting Franck-Condon Shifts from Thermally Broadened Photoneutralization Cross-Section Bands of Deep Traps in Semiconductors

Physica Status Solidi. B: Basic Research. - 186. 1994, 2, K63

- K68 


\title{
Alternative Way for Detecting Franck-Condon Shifts from Thermally Broadened Photoneutralization Cross-Section Bands of Deep Traps in Semiconductors
}

\author{
By \\ R. PÄSSLER
}

Basic optical and thermal parameters of deep traps in semiconductors usually are represented by corresponding configuration co-ordinate diagrams (Fig. 1). Such diagrams are characterized above all by the non-vertical separations $J_{\mathrm{n}} \equiv E_{\mathrm{c}}-E_{\mathrm{t}}$ and $J_{\mathrm{p}} \equiv E_{\mathrm{t}}-E_{\mathrm{v}}$ between the minima $E_{\mathrm{c} / \mathrm{v}}$ and $E_{\mathrm{t}}$ of the relevant electronic energy + lattice potential energy curves in the alternative charge state conditions "empty" (= trapped hole (p)) and "full" (= trapped electron (n)). Another crucial parameter determining the mutual constellations of such CC-curves is the lattice adjustment (relaxation) energy $A[1,2]$. This represents the potential energy taken by the lattice due to elongations of lattice oscillator co-ordinates $Q$ between their alternative equilibrium positions $Q_{\mathrm{p}}$ and $Q_{\mathrm{n}}$ (cf. Fig. 1). In terms of these fundamental

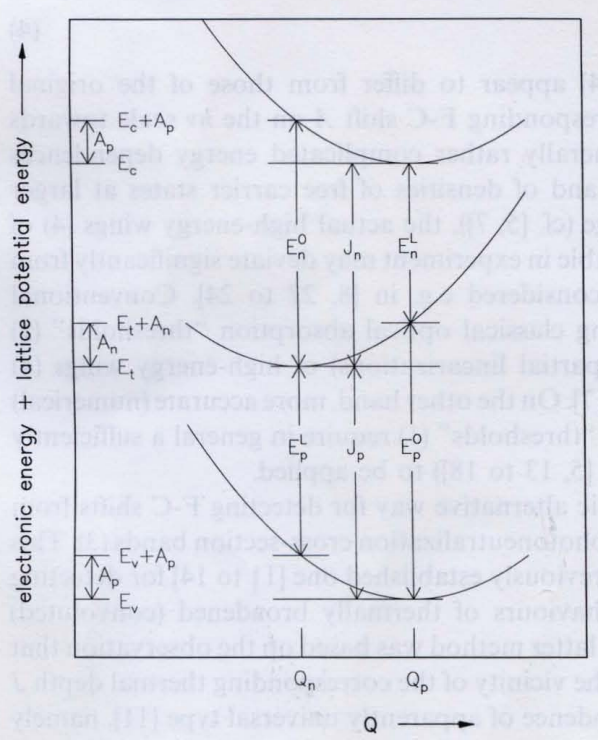
trap parameters [2] the classical optical absorption "thresholds" are well known [2 to 8 ] to be given by

$$
E_{\mathrm{n} / \mathbf{p}}^{\mathbf{O}}=J_{\mathrm{n} / \mathrm{p}}+A
$$

and the centres of gravity of the associated luminescence bands [2 to 4] (if observable [9]) are located at

$$
E_{\mathrm{n} / \mathrm{p}}^{\mathrm{L}}=J_{\mathrm{n} / \mathrm{p}}-A .
$$

Thus the lattice relaxation energy $A$ corresponds just to the magnitude of the charac-

Fig. 1. Configuration co-ordinate diagram of a deep trap for the commonly considered regime of equality $\left(A_{\mathrm{p} / \mathrm{n}}=A\right)$ of the lattice adjustment energies $A_{\mathrm{p}}$ and $A_{\mathrm{n}}$ in the alternative charge state conditions "empty" (p) and "full" (n)

\footnotetext{
1) D-09107 Chemnitz, Federal Republic of Germany.
} 
teristic shift of the classical absorption "threshold" (1) or the luminescence-band centre of gravity position (2) with respect to the associated zero-phonon energy position ("thermal depth") $J_{\mathrm{n} / \mathrm{p}}$ [2, 10 to 14], which is commonly referred to as Franck-Condon shift ( $A \equiv d_{\text {F-C }}=S \hbar \omega$ [15 to 18$]$ ). A convenient way for determining this basic trap parameter $A$ is well known from standard literature to be based on evaluations of low-order moments of luminescence bands due to radiative capture of thermal carriers into the corresponding trap (see e.g. [10, 19 to 21]). In such cases the parameters $J_{\mathrm{n} / \mathrm{p}}$ and $A$ can be detected from first moments (centres of gravity) in combination with second moments (effective widths) with relatively high degree of accuracy due the fact that there is only a relatively narrow band-edge region (of order $k T$ ) involved in the corresponding luminescence processes. Unfortunately, it is matter of principle that such an assessment procedure applies exclusively to radiative (luminescence) centres. In other words, no information on magnitudes of lattice relaxation energies can be obtained in such a way in cases of non-radiative (fast recombination) centres, where no luminescence band is detectable.

There has been suggested, among other things in literature, also a certain way for estimating the magnitudes of lattice relaxation energies $A$ (F-C shifts) in connection with graphical detections of classical optical absorption "thresholds" $J_{\mathrm{n} / \mathrm{p}}+A$ (1) (cf. e.g. [5 to $8,22,23])$. Such procedures are essentially based on theoretical observations for the corresponding thermally broadened (convoluted) photoexcitation cross-sections of type

$$
\sigma_{\mathbf{n} / \mathbf{p}}(h v, T)=\int \mathrm{d}(h \tilde{v}) \tilde{\sigma}_{\mathbf{n} / \mathbf{p}}(h \tilde{v}) R(h v-h \tilde{v}, T)
$$

$[5,10$ to 14$]$ at sufficiently high photon energies $\left(h v \gg J_{\mathbf{n} / \mathbf{p}}+A\right)$ to approach closely the approximately $T$-independent asymptotes [8],

$$
\sigma_{\mathrm{n} / \mathbf{p}}(h v, T) \rightarrow \tilde{\sigma}_{\mathbf{n} / \mathbf{p}}(h v-A) .
$$

Thus the convoluted high-energy wings (4) appear to differ from those of the original electronic parts $\tilde{\sigma}_{\mathrm{n} / \mathrm{p}}(h v)$ in (3) but by a corresponding F-C shift $A$ on the $h v$ scale towards higher energies. Unfortunately, due to generally rather complicated energy dependences both of dipole transition matrix elements and of densities of free carrier states at larger distances from the corresponding band edge (cf. [5, 7]), the actual high-energy wings (4) of photoexcitation cross-section bands observable in experiment may deviate significantly from simple analytical dependences like those considered e.g. in [8, 22 to 24]. Conventional procedures like $[5,6,8,22,23]$ for detecting classical optical absorption "thresholds" (1) by means of convenient transformations (partial linearizations) of high-energy wings (4) may thus involve considerable uncertainties [7]. On the other hand, more accurate (numerical) determinations of such classical absorption "thresholds" (1) require in general a sufficiently elaborate computational apparatus (cf. e.g. [5, 13 to 18]) to be applied.

It is the aim of this note to present a basic alternative way for detecting F-C shifts from experimentally observed $h v$ dependences of photoneutralization cross-section bands (3). This new method is closely connected with our previously established one [11 to 14] for detecting thermal depths $J$ from low-energy tail behaviours of thermally broadened (convoluted) photoexcitation cross-section bands (3). The latter method was based on the observation that the $h v$ dependence of a $\sigma(h v, T)$ curve (3) in the vicinity of the corresponding thermal depth $J$ is closely approaching an exponential dependence of apparently universal type [11], namely

$$
\sigma(h v, T) \propto \exp \left(\frac{h v}{2 k T}\right) \text { at } h v \approx J .
$$


Removing this common slope (5) by a transformation of the form [11 to 14]

$$
\varrho(h v, T) \equiv \exp \left(-\frac{h v}{2 k T}\right) \sigma(h v, T)
$$

we obtained an auxiliary function the maximum of which was located in the immediate vicinity of $J$ (at some few $k T$ above $J$ ). Particularly with respect to processes of photoneutralization [11, 13] being allowed in dipole approximation we have shown in [13] that the residual distance of the maximum $h v^{(\varrho)}$ of a $\varrho$-curve (6) with respect to $J$ is approximately equal to [11]

$$
h v^{(e)}-J \rightarrow 2 k T \text {. }
$$

In this way we could readily detect thermal depth positions $J$ from empirical maximum positions $h v^{(\varrho)}$ of transformed photoneutralization curves $\varrho(h v, T)$ of type (6) (cf. also Fig. 2 and 3 of the present note).

Now, in addition to (5), we have found and confirmed by a series of theoretical investigations (unpublished so far) that there is still another characteristic point (2) on the $h v$ scale where the $h v$ dependence of a given $\sigma(h v, T)$ curve (3) approaches again some exponential dependence of universal type, namely

$$
\sigma(h v, T) \propto \exp \left(\frac{h v}{k T}\right) \text { at } h v \approx J-A .
$$

Thus, in analogy to (6), it is possible again to remove this common slope (8) by a transformation of type

$$
\pi(h v, T) \equiv \exp \left(-\frac{h v}{k T}\right) \sigma(h v, T),
$$

so that the maximum of the latter curve should be located in the immediate vicinity of the characteristic point $J-A(2)$. Particularly with respect to processes of photoneutralization being allowed in dipole approximation we have performed detailed analytical and numerical investigations of characteristic $h v$ dependences of $\pi$-transforms (9) (in analogy with our previous study [13] of characteristic $h v$ dependences of $\varrho$-transforms (6)). In this way we have found the residual distance of the maximum $h v^{(\pi)}$ of a $\pi$-curve (9) with respect to $J-A(2)$ to reduce approximately to

$$
h v^{(\pi)}-(J-A) \rightarrow k T .
$$

Combining finally (7) with (10) we see that the F-C shift in question is represented approximately by

$$
A \rightarrow h v^{(\varrho)}-h v^{(\pi)}-k T .
$$

This gives us the possibility of detecting magnitudes of F-C shifts in a simple way from the mutual distances of maximum positions $h v^{(\varrho)}$ and $h v^{(\pi)}$ of the associated auxiliary curves $\varrho(h v, T)(6)$ and $\pi(h v, T)(9)$ (provided, of course, that the experimental measurements of photoneutralization curves $\sigma(h v, T)$ are comprehensive enough for a clear exhibition of the maxima of both auxiliary curves in the corresponding $h v$ regions). 


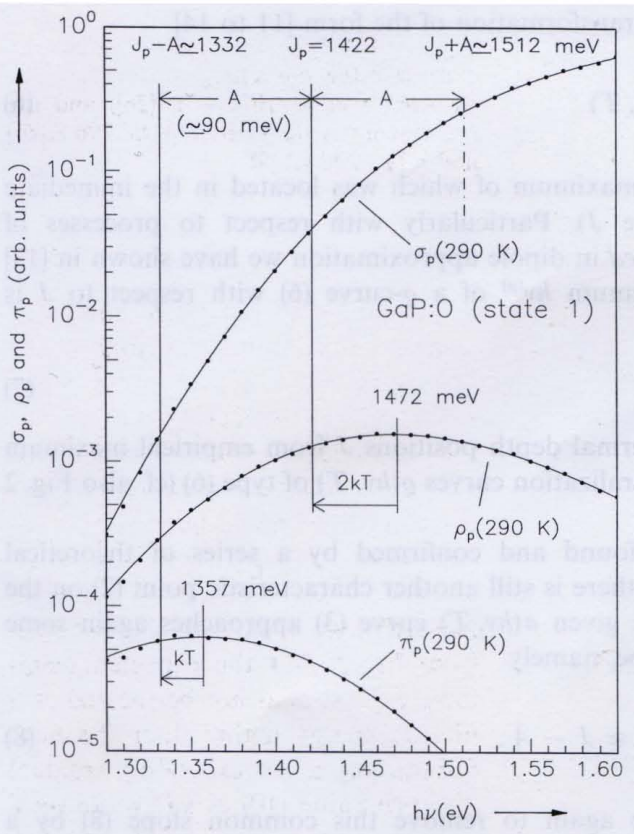

Fig. 2. Experimental hole photoexcitation cross-section band $\sigma_{\mathrm{p}}(h v, 290 \mathrm{~K})$ of the oxygen donor level (state 1) in $\mathrm{GaP}$ given by Samuelson and Monemar (Fig. 3 in [25]) and the associated auxiliary curves $\varrho_{\mathrm{p}}(h v$, $290 \mathrm{~K})(6)$ and $\pi_{\mathrm{p}}(h v, 290 \mathrm{~K})(9)$

Let us test this novel method here first of all by reconsidering the case [11] of optical hole excitation from the oxygen donor level (state 1) in GaP which has been studied both experimentally and theoretically in great detail by Monemar and Samuelson [10, 25]. Inserting in particular their $\sigma_{\mathrm{p}}(h v, 290 \mathrm{~K})$ data (Fig. 3 in [25]) into (6) we find a maximum position $h v^{(\varrho)}$ at about $1472 \mathrm{meV}$ (Fig. 2). Subtracting the corresponding residual distance value (7) of $2 k T=50 \mathrm{meV}$ we attain again a thermal depth position of $J_{\mathrm{p}}(290 \mathrm{~K})$ $=1422 \mathrm{meV}$ (in agreement with [11]). Inserting, alternatively, these experimental $\sigma_{\mathrm{p}}(h v, 290 \mathrm{~K})$ data into (9) we find a maximum position $h v^{(\pi)}$ located at about $1357 \mathrm{meV}$ (Fig. 2). Subtracting, accordingly, the corresponding residual distance value (10) of $k T=25 \mathrm{meV}$ we attain a characteristic position (2) of $J_{\mathrm{p}}(290 \mathrm{~K})-A=1332 \mathrm{meV}$. Taking finally the distance between both characteristic points $J_{\mathrm{p}}$ and $J_{\mathrm{p}}-A$ we come thus to a F.-C. shift value $A$ of about $90( \pm 5) \mathrm{meV}$, which may be compared with an earlier value of $85( \pm 5) \mathrm{meV}$ due to Monemar and Samuelson's elaborate deconvolution procedure $[10,25]$. Moreover, the present F-C shift value of $90 \mathrm{meV}$ is just coincident with that detected by Kopylov and Pikhtin's semiclassical analysis [15]. The approximate equality between these three numerical F-C shift values due to different authors may be taken as a confirmation of a sufficiently high degree of adequacy and compatibility of their basically different methods of quantitative analyses. At the same time this comparison shows that it should be possible to detect correct orders of magnitude for F-C shifts in a simpler (computationally much less expensive) way than in $[10,19,25]$ or [15].

As a further instructive example let us consider the photoconductivity data given by Amato et al. (Fig. 4 in [26]) for GaAs: Cr. In analogy with [5, 26] we do not consider here the eventuality of an occurrence of Sommerfeld (Coulomb) factor effects [14], i.e. we associate the given photoconductivity curve $\propto \sigma$ (hv, 296 K) (Fig. 4 in [26] reproduced here in Fig. 3) with the model of a neutralized centre [13]. (Note that this assumption is convenient in particular for a direct comparison of the present F-C shift value with the earlier one given in $[5,26]$.) The maximum position $h v^{(\varrho)}$ of the associated $\varrho(h v, 296 \mathrm{~K})$ curve (6) (in Fig. 3) is seen to be located at about $764( \pm 10) \mathrm{meV}$. Subtracting a corresponding residual distance value (7) of $2 k T=51 \mathrm{meV}$ we come to an approximate thermal depth value of $J(296 \mathrm{~K})=713$ 


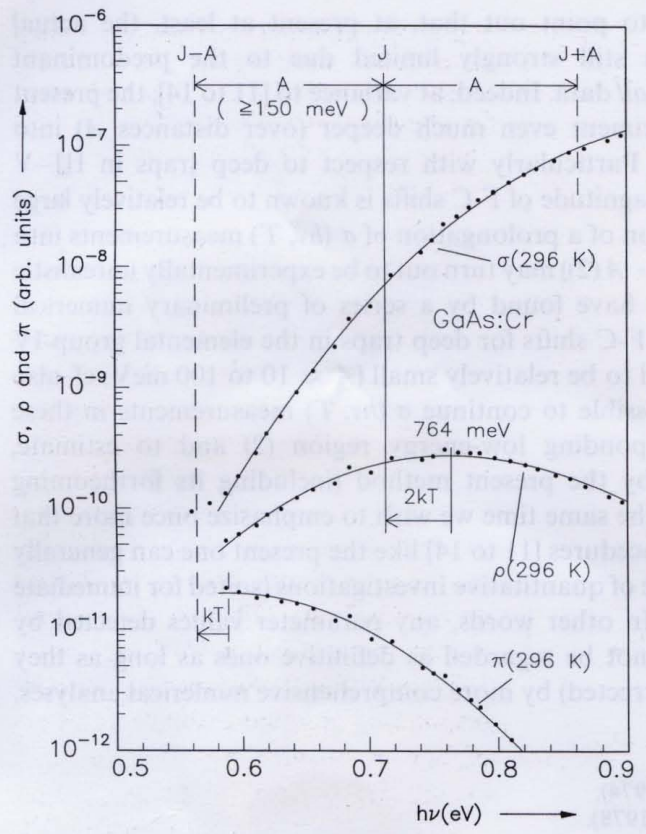

Fig. 3. Experimental photoconductivity curve $\propto \sigma(h v, 296 \mathrm{~K})$ as given for $\mathrm{GaAs}: \mathrm{Cr}$ by Amato et al. (Fig. 4 in [26]) and the associated auxiliary curves $\varrho(h v, 296 \mathrm{~K})(6)$ and $\pi(h v, 296 \mathrm{~K})(9)$

$( \pm 10) \mathrm{meV}$. On the other hand, the expected maximum behaviour of the associated $\pi(h v, 296 \mathrm{~K})$ curve (9) (in Fig. 3) cannot be clearly identified due to rather strong deviations of the lowest experimental points from their theoretically expected positions in connection with a complete lack of further low-energy tail data ( $h v \leqq 550 \mathrm{meV})$. Nevertheless, we can conclude at least from Fig. 3 that the expected maximum $h v^{(\pi)}$ should not be located at a photon energy higher than $588 \mathrm{meV}$. Subtracting a corresponding residual distance value (10) of $k T=25 \mathrm{meV}$ we come thus to an upper bound estimation of $J(296 \mathrm{~K})-A \leqq 563 \mathrm{meV}$

for the characteristic point (2). In this way the F-C shift in question is found to be confined at least by a lower bound of about $150 \mathrm{meV}$, i.e. $A \geqq 150 \mathrm{meV}$. Such an F-C shift value is, above all, considerably larger than an earlier one of only $90 \mathrm{meV}[5,26]$. (Note that this earlier underestimation was obviously due to the relative roughness of the corresponding approach in $[5,26]$.) In this connection it may be of interest to refer still to detailed numerical analyses $[20,21]$ of luminescence bands associated with deep $\mathrm{GaAs}$ : $\mathrm{Cr}$ levels which have given throughout F-C shift values of the order of $A=170$ to $200 \mathrm{meV}$. Thus the present lower bound estimation of $A \geqq 150 \mathrm{meV}$ (Fig. 3) is consistent (relatively closely approaching) with this typical magnitude of F-C shift values for nearly midgap GaAs : Cr levels [20, 21].

In concluding this note we wish to emphasize that, in complete analogy to our preceding papers [11 to 14], the present results are valid only under the basic assumptions [12] that

1. there is no transition-induced phonon energy shift (represented here in Fig. 1 by the assumed equality $A_{\mathrm{n}}=A_{\mathrm{p}}$ of the lattice adjustment energies in both alternative charge state conditions) and

2. there is no other broadening mechanism in competition with the MP mechanism considered (excluding above all electric field broadening effects; cf. [17]).

Moreover, in analogy to $[11,13]$, the characteristic residual distance values (7) and (10) apply only to processes of photoneutralization that are allowed in dipole approximation (near the band edge, at least). A generalization of the present F-C shift detection method for processes of photoionization at charged centres (particularly repulsive ones) can be easily performed in anlogy to that for our preceding thermal depth detection method [14]. In this way the field of possible applicability of this novel method may be considerably 
enlarged. At the same time we wish to point out that, at present at least, the actual applicability of this novel method is still strongly limited due to the predominant non-availability of corresponding band tail data. Indeed, at variance to [11 to 14], the present method requires to penetrate in experiment even much deeper (over distances $A$ ) into lower-energy tail regions (cf. also [5]). Particularly with respect to deep traps in III-V compounds where the typical order of magnitude of F-C shifts is known to be relatively large ( $A \approx 100$ to $300 \mathrm{meV}$ [17]), the suggestion of a prolongation of $\sigma(h v, T)$ measurements into the relevant low-energy region ( $h v \rightarrow J-A(2))$ may turn out to be experimentally unrealistic in many cases. At variance to this we have found by a series of preliminary numerical investigations (unpublished so far) that $\mathrm{F}$-C shifts for deep traps in the elemental group-IV semiconductor materials $\mathrm{Si}$ and $\mathrm{Ge}$ used to be relatively small ( $A \approx 10$ to $100 \mathrm{meV}$; cf. also [27]). Thus it should be more easily possible to continue $\sigma(h v, T)$ measurements in these materials downwards into the corresponding low-energy region (2) and to estimate, henceforth, magnitudes of F-C shifts by the present method (including its forthcoming generalization for charged centres). At the same time we wish to emphasize once more that semiempirical (graphical) assessment procedures [11 to 14] like the present one can generally be considered only as a preliminary stage of quantitative investigations (suited for immediate realizations in experimental practice). In other words, any parameter values detected by such semiempirical procedures should not be regarded as definitive ones as long as they are not clearly confirmed (or slightly corrected) by more comprehensive numerical analyses.

\section{References}

[1] R. Pässler, Czech. J. Phys. B24, 322 (1974).

[2] R. Pässler, phys. stat. sol. (b) 85, 203 (1978).

[3] M. Lax, J. chem. Phys. 20, 1752 (1952).

[4] D. Curie, Luminescence in Crystals, Methuen \& Co. Ltd., London and J. Wiley \& Sons Inc., New York 1963.

[5] B. K. Ridley and M. A. Amato, J. Phys. C 14, 1255 (1981).

[6] J. M. Noras, J. Phys. C 14, L713 (1981).

[7] B. K. Ridley, J. Phys. C 14, L717 (1981).

[8] R. PÄSSler, phys. stat. sol. (b) 162, K47 (1990).

[9] R. PÄsSLER, phys. stat. sol. (b) 65, 561 (1975).

[10] B. Monemar and L. Samuelson, Phys. Rev. B 18, 809 (1978).

[11] R. PÄsSLER, phys. stat. sol. (b) 158, K143 (1990).

[12] R. PÄSSLER, phys. stat. sol. (b) 167, 165 (1991).

[13] R. PÄSSLER, phys. stat. sol. (b) 170, 219 (1992).

[14] R. PÄSSLER, phys. stat. sol. (b) 179, 133 (1993).

[15] A. A. Kopylov and A. N. Pikhtin, Fiz. Tekh. Poluprov. 8, 2398 (1974).

[16] A. Chantre, G. Vincent, and D. Bois, Phys. Rev. B 23, 5335 (1981).

[17] G. F. Neumark and K. Kosai, Semiconductors and Semimetals 19, 1 (1983).

[18] S. Loualiche, A. Nouailhat, G. Guillot, and M. Lanoo, Phys. Rev. B 30, 5822 (1984).

[19] B. Monemar and L. Samuelson, J. Lum. 12/13, 507 (1976).

[20] P. Leyral, F. Litty, S. Loualiche, A. Nouailhat, and G. Guillot, Solid State Commun. 38, 333 (1981).

[21] A. Nouailhat, F. Litty, S. Loualiche, P. Leyral, and G. Guillot, J. Physique 43, 815 (1982).

[22] J. M. Noras and J. W. Allen, J. Phys. C 13, 3511 (1980).

[23] S. Fung and R. J. Nicholas, J. Phys. C 14, 2135 (1981).

[24] J. C. Inkson, J. Phys. C 14, 1093 (1981).

[25] L. Samuelson and B. Monemar, Phys. Rev. B 18, 830 (1978).

[26] M. A. Amato, M. C. Arikan, and B. K. Ridley, Proc. Semi-Insulating III-V Materials Conf., Nottingham 1980, Ed. G. J. ReEs (p. 249).

[27] H. Pettersson, H. G. Grimmeiss, K. Schmalz, A. Knecht, and R. PÄssler, submitted to J. appl. Phys. (1994). 